\title{
Balancing operating theatre and bed capacity in a cardiothoracic centre
}

\section{John Bowers}

Stirling Management School, University of Stirling, Stirling FK9 4LA, United Kingdom

e-mail address: j.a.bowers@stir.ac.uk

Tel: +44 1786 467377; fax: +44 1786467329

\begin{abstract}
This paper examines the balance between operating theatre and bed capacity in a specialist facility providing elective heart and lung surgery. The capacity of the whole facility is determined by the availability of operating theatre time and Intensive Care beds: without both resources surgery has to be postponed. Although the admissions can be managed, there are significant stochastic components, notably the cancellation of theatre procedures and patients' length of stay on the Intensive Care Unit. A simulation was constructed to explore the interdependencies of resource availabilities and the daily demand. The model was developed with clinical and management staff to explore options for expanding the capacity of the whole facility. Ideally the bed and theatre capacity should be well balanced but unmatched increases in the capacity of either resource can still be beneficial. The study provides an example of a capacity planning problem in which there is uncertainty in the demand and availabilities of two symbiotic resources.
\end{abstract}

Keywords: OR in health services; intensive care; staff scheduling; simulation

\section{Background}

The Heart and Lung Centre (HLC) at the Golden Jubilee National Hospital was established in 2008 as specialist National Health Service (NHS) facility for cardiac and thoracic surgery. A key component is the Intensive Care Unit (ICU) which is essential for many patients undergoing the specialist procedures at the HLC. A distinguishing feature of this ICU is that the large majority of the activity is elective: a patient's surgery can only proceed if there is both operating theatre time and an ICU bed available otherwise it is postponed to another day. Capacity planning for surgical specialties often focuses on the operating theatres: the beds are regarded as a secondary resource requirement that seldom constrains the overall capacity (Guerriero, 2011). However, capacity in a facility such as the HLC depends on the balance of operating theatre and ICU bed availability. The formation of the HLC involved a transfer of staff and resources from a variety of hospitals, with a reorganisation of working practices. The new centre was unable to meet demand, resulting in significant waiting lists; this study examined the options for enhancing the capacity of the HLC. 
Cardiac and thoracic surgery have a high profile and their waiting lists attract particular attention. However, the services are also expensive, especially since many patients require an ICU stay involving 24 hour care from a dedicated nurse. Given typical working patterns this implies 5.9 nurses for every bed though this ratio varies depending on local conditions and the physical layout of the ICU. In addition to the dedicated ICU nurse the need for other support and supervisory staff, and specialised equipment all add to the cost of care in an ICU.

\section{Modelling ICU's and the balance of resources}

The need for rigorous analysis when planning Intensive or Critical Care Units is reflected in the many operational research studies of subject. Most ICU's have to cope with a large stochastic demand generated by emergency admissions, and simulation is often employed as the prime analytical tool (Kim, 1999; Kim \& Horowitz, 2000; Ridge et al., 1998; Kolker, 2009; Griffiths et al., 2005; Mallor \& Azcárate, 2011). Even though some patients may have undergone elective surgery, their ICU stay is often not anticipated: the nature of their surgery may imply the chance of an ICU stay but effectively this flow of patients is modelled as stochastic, though with a weekly mean arrival pattern reflecting typical theatre activity (Griffiths et al., 2005; Griffiths et al., 2010; Shahani et al., 2008). Other studies (Vanberkel et al., 2010) may assume that surgery will not be postponed due to a lack of a bed but this is a very real possibility in the HLC. In the HLC, the elective surgery is characterised by a very high proportion of the patients requiring at least a short ICU stay: a surgical procedure will only begin if an ICU bed is available. While long delays would not be acceptable, surgery can often be postponed for a few days providing some scope to manage admissions. Synchronising the use of operating theatre time and ICU beds is a major organisational challenge at the HLC: both are expensive (ISD Scotland, 2011) and need to be well managed. Although much of the HLC activity can be planned, theatre procedures may be cancelled for a variety of reasons and a patient's length of stay on the ICU is highly variable and unpredictable. This introduces a significant stochastic element and adds to the management challenge.

Capacity planning studies often consider the trade-off between service level and the utilisation of one dominant resource, as when considering ICU bed provision and the implications for rejecting or diverting patients (Kim, 1999). Many models do not include crucial management responses to demand; in reality there may be opportunities to manage admissions, as in the HLC, or expedite patient transfer or discharge of patients. Ignoring such management responses can lead to overestimates of the capacity requirements (Costa et al., 2003). In some units, there may be the possibility to expedite the transfer of some patients out of the ICU if occupancy levels are high, or extending the stay when occupancy is low (Mallor \& Azcárate, 2011). Such responses were not identified at the HLC, though further investigation could be useful. Another study incorporating management responses considered the possibility of revising the schedules of the operating theatres feeding the ICU (Kolker, 2009), producing a smoother flow of activity that improved the service levels, assuming a fixed bed capacity. The HLC has to manage both ICU bed stay and operating theatre time, striving to achieve a balance between the utilisation of these expensive, scarce resources and the overall service level. 


\section{Referrals and Length of stay}

A key component of demand for the HLC was the number of referrals for surgery. However, estimates of elective referrals require some caution and simply using historic admissions to the HLC as a measure of demand can be misleading; treatment may be postponed either as a result of a lack of capacity or the personal characteristics and preferences of the patient. This problem can be avoided by using longer time series but changes in practice may cast some doubt about the relevance of older data. The problem was aggravated at the HLC since it had only been operational for a relatively short time and referral practices were still evolving. At the time of the study, ICU admissions were typically 25-30 per week, with $90 \%$ coming from cardiac surgery. Increases in some waiting lists suggested that additional capacity was required. The data quoted in throughout this paper should be regarded as illustrative of an early phase of the development of the new HLC; practice has evolved and the data do not necessarily reflect current experience at the HLC.

The other component of demand for the ICU is the length of stay. This is highly variable, as illustrated by the empirical distribution of patients' ICU stay in Fig.1 based on 758 observations; many patients just spend 1 day in an ICU bed but stays of 2-5 days are common and a few patients require 10-50 days in the ICU. Similar distributions have been noted in other ICU's, particularly where the admissions include elective patients (McManus et al., 2004; Griffiths et al., 2005). Hyperexponential distributions have been successfully employed in modelling length of stay in diverse healthcare systems ranging from labour wards (Harper et al, 2012) to geriatrics (McClean \& Millard, 2003). The simplest form is the order 1 exponential distribution (Litvak et al., 2008) and this provides an approximate but imperfect fit to the HLC length of stay data, as illustrated in Fig.1. However, it has been suggested that while an exponential distribution can provide a reasonable fit for emergency ICU stay it is not appropriate for elective patients (Kim, 1999). In the current application, an order 2 hyperexponential model offers some improvement but an order 3 model provides a significantly better fit, as illustrated in Fig.1. The best fit parameters for the proportions and negative exponential coefficients are included in Table 1. This length of stay model suggests three categories of patients, relating well to clinicians' practical distinctions (Vasilakis \& Marshall, 2005). The patients in the third category deserve particular attention: the relatively high probability of a long stay may well be the result of a combination of particularly difficult surgery and co-morbidity. The potential of a long stay on the ICU may be inevitable for some patients but perhaps others might benefit from having their surgery postponed until they are in a medical state that might reduce the chance of such a long stay. 


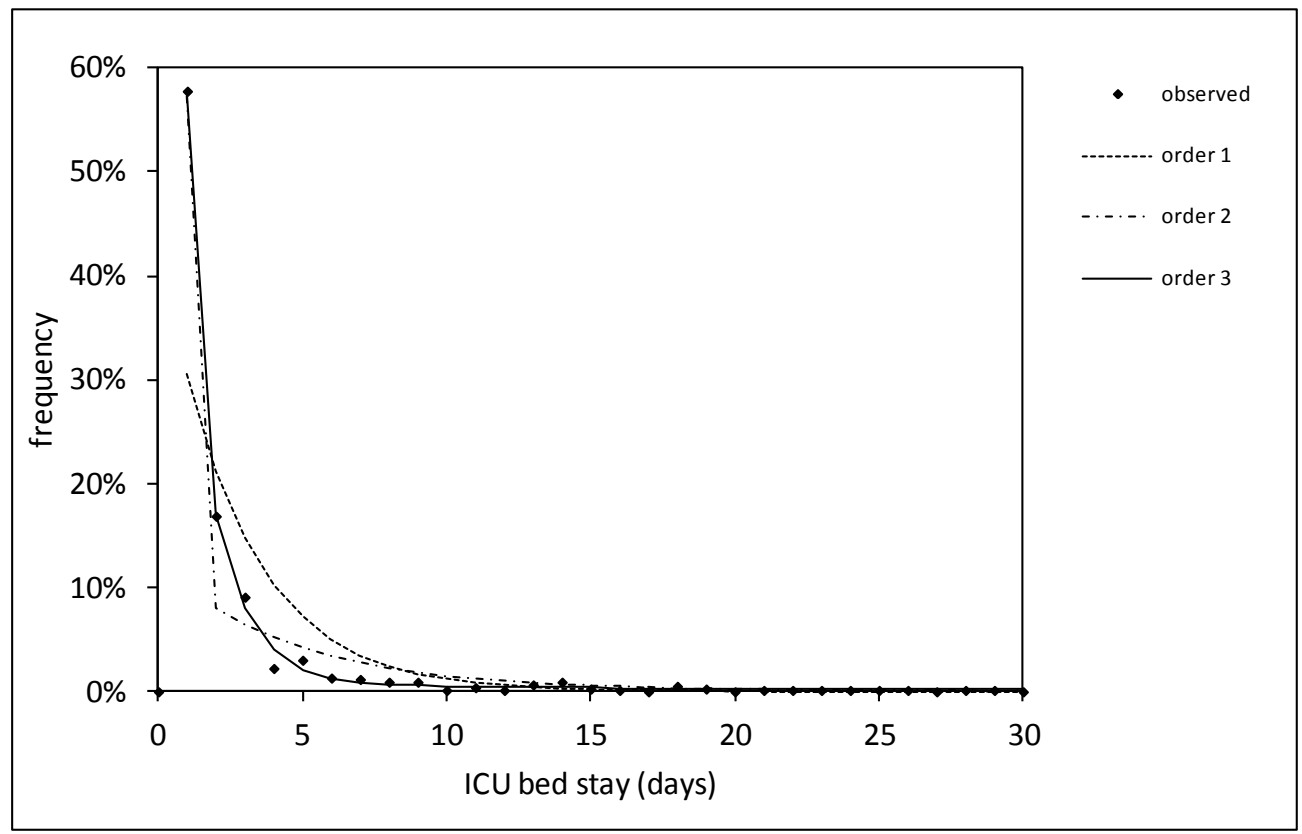

Fig.1 Fitting a length of stay model

Table 1 Best fit parameters for an order 3 hyperexponential length of stay model

\begin{tabular}{lccc}
\hline patient category & proportion $=l_{j}$ & negexp coef $=v_{j}$ & mean LoS (nights) \\
\hline 1. one night stay & $21.0 \%$ & - & 1.00 \\
2. medium stay & $65.7 \%$ & 0.46 & 1.83 \\
3. long stay & $13.3 \%$ & 0.95 & 17.10 \\
\hline all ICU admissions & $100.0 \%$ & - & 3.69 \\
\hline
\end{tabular}

Some studies have noted a correlation between the ICU length of stay and the severity of a patient's condition, as measured by the logistic organ dysfunction score (LODS) (Heldwein et al., 2011). However, this relationship appears valid for just specific categories of patients (Mallor \& Azcárate, 2011). The observed correlation at the HLC was weak at -0.09 ; this was insufficient to justify developing an admissions' system using LODS to predict ICU stay and select elective patients and help balance the operating theatre time and ICU bed utilisation. Severity scoring systems may have clinical value, their use as management tools can be problematic (Sherck \& Shatney, 2011). 


\section{Developing the simulation}

The HLC simulation model was developed using Simul8; although the model focussed on the interactions between the operating theatres and the ICU, these were placed in the context of the whole patient journey from the "decision to treat" to discharge from the ward, as illustrated in Fig.2. While the model can accommodate deviations from the common patient journey through the HLC, the large majority of patients requiring an ICU stay are elective and they follow the sequence of a scheduled operating theatre procedure, a short ICU stay, transfer to the High Dependency Unit (HDU) and then on to a conventional ward before discharge from the hospital. Preliminary analysis indicated that the major constraints on the HLC's capacity were the operating theatre and ICU bed availability, hence these became the focus of the simulation. The HLC had access to a number of operating theatres; elective surgery was usually restricted to Monday-Friday but the model was developed to explore a range of working practices, including weekend surgery. The operating theatre capacity was usually defined by the number of patients per day: the specialised procedures were long and complex so an operating theatre session was usually dedicated to a single patient. Given the nature of the surgery, the medical condition of the patients is most important and postponements, or cancellations due to organisational difficulties, were relatively common. In estimating the inherent theatre cancellation rate it was important to distinguish the causes, excluding those cancellations due to a lack of an ICU bed. The theatre cancellation rate was then embedded in the simulation as a simple binomial model of completed procedures, and hence admissions on to the ICU given the daily theatre schedule.

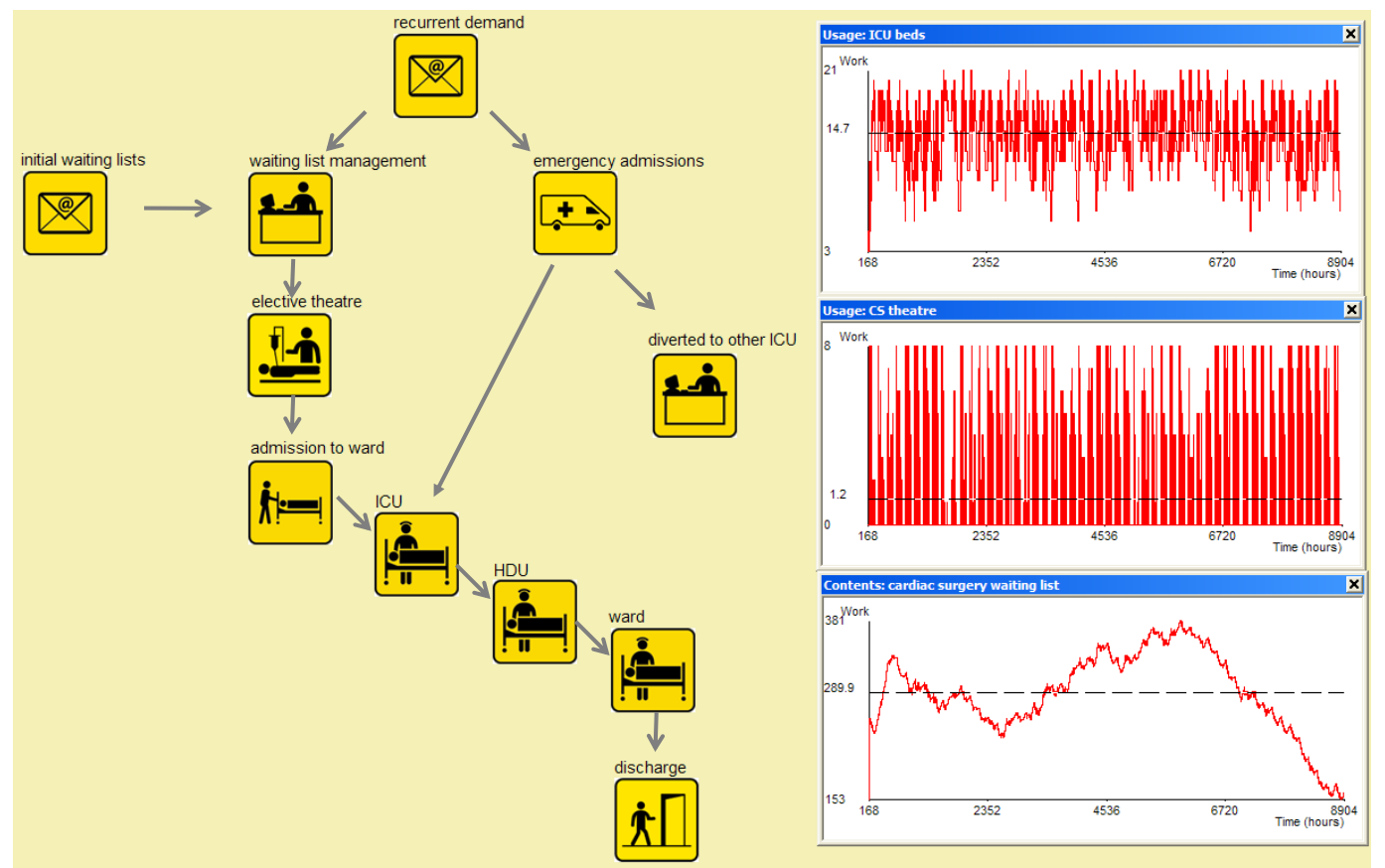

Fig.2 Heart and Lung Centre simulation

While the capacity of the ICU was specified in terms of beds, this was actually determined by the staff availability. The ICU typically had spare bed capacity, providing some short term flexibility and also scope for future expansion of the HLC. The simulation allowed the user to specify the ICU capacity as a staffing 
profile over the week and also the daily theatre schedule. At the start of each simulated day, the bed availability in the ICU is checked before allocating patients to particular theatre sessions: some theatre sessions will be unused if no ICU bed is available. The key simulation outputs were the number of admissions and the utilisation of the theatres and ICU beds; comparisons of these metrics with historic data helped validate the model and confirm its value as a basis for decision making. Clinical and management staff were very active in the development of the simulation and used it to explore various organisational issues, helping to identify cost effective options for enhancing the HLC capacity.

\section{Staffing and ICU capacity}

The simulation model provided a mechanism for assessing the service level implications, given the specified demand and the available resources. However, any estimate of the HLC capacity depended on both the component resource capacities and their management. In particular the assessment depended on assumptions about the deployment of the available ICU staff. Stochastic mathematical programming might be used to determine the optimal allocation of staff (Morton \& Popova, 2004). However, experiments indicated that in the present example a relatively simple staff allocation was sufficient based on the minimisation of an approximation of the expected excess ICU requirement $X$, given a specified pattern of theatre activity:

$$
\mathrm{E}[X]=\sum_{i=1}^{7} \sum_{d=b_{i}}^{\infty}\left(d-b_{i}\right) P_{i}(d)
$$

where:

$w \quad=$ ward capacity (maximum number of beds available on any one

$$
b_{i} \quad=\text { ICU bed capacity on day } i \text { determined by staffing; } b_{i} \leq w(i=
$$

staffing

$$
\mathbf{B}=\sum_{i=1}^{7} \boldsymbol{b}_{i} \text { since budgetary constraints limit the weekly }
$$

$\overline{d_{i}} \quad=$ mean ICU bed demand on day $i$, reflecting the theatre schedule, cancellation rate and ICU length of stay distribution

$P_{i}(d)=$ probability that bed demand on day $i$ is $d$, assuming a Poisson distribution with a mean demand for day $i$ of $\overline{d_{i}}$

While the total staffed bed capacity $B$ was constrained by the budget, the number available on any one day was also limited, though in this application this was not a significant constraint. $\bar{d}_{i}$ was estimated assuming that the contributions from the three components of demand identified in Table 1 are independent with $\bar{d}_{i}(j)$ being the mean demand on day $i$ for component $j$ of demand $(j=1 . .3)$. Other studies (Adan et al., 2011) have formulated similar problems but adopted more complex distributions of ICU bed-stay. However, the exponential nature of the 
length of stay model permits a comparatively simple formulation reflecting the remaining patients from previous days' admissions to the ICU and the 7-day cycle of activity. Each of the three hyperexponential components $(j=1 . .3)$ of Table 1 , contributes to the mean bed demand on day $i(i=1 . .7)$ reflecting the daily theatre capacity $t_{i}$ specified by the week's schedule:

$$
\bar{d}_{i}(j)=l_{j}\left(t_{i}(1-c)+t_{(i-1) \bmod 7}(1-c) v_{j}+t_{(i-2) \bmod 7}(1-c) v_{j}^{2}+\ldots\right)
$$

\section{(2)}

where:

$t_{i} \quad=$ available theatre capacity (number of patients) on day $i$

$c \quad=$ theatre cancellation rate

$l_{j} \quad=$ proportion of patients of category $j$, see Table 1

$v_{j} \quad=$ fitted exponential parameter corresponding to the mean stay for category $j$ patients, see Table 1 .

A high theatre cancellation rate can have a large effect on the demand for beds, especially for the long stay category of patients, $j=3$. In the current study there was no significant relationship distinguishing the cancellation rates for different categories, though this could be included if required. Exploiting the common summations applicable to the geometric nature of the model of accumulating bed demand, this can be rewritten as:

$$
\begin{aligned}
& \bar{d}_{i}(j)=l_{j} \sum_{k=0}^{6} t_{(i-k) \bmod 7}(1-c) v_{j}^{k}+l_{j} v_{j}^{7} \sum_{k=0}^{6} t_{(i-k) \bmod 7}(1-c) v_{j}^{k}+l_{j} v_{j}^{14} \sum_{k=0}^{6} t_{(i-k) \bmod 7}(1-c) v_{j}^{k}+\ldots \\
& \bar{d}_{i}(j)=\frac{l_{j} \sum_{k=0}^{6} t_{(i-k) \bmod 7}(1-c) v_{j}^{k}}{1-v_{j}^{7}}
\end{aligned}
$$

Hence considering the sum of the components of the order 3 hyperexponential length of stay model:

$$
\overline{d_{i}}=\sum_{j=1}^{3} \frac{l_{j}}{1-v_{j}^{7}} \sum_{k=0}^{6} t_{(i-k) \bmod 7}(1-c) v_{j}^{k}
$$

Using this estimate of mean daily demand to specify a Poisson distribution of demand in Eq.1, $\mathrm{E}[X]$ was minimised using a standard branch and bound integer programming routine to estimate the optimal staffing profile described by $b_{i}$ ( $i$ $=1 . .7)$ for a specified theatre allocation $t_{i}(i=1 . .7)$.

The assumptions used in determining these staffing profiles were tested in a series of experiments using the simulation. Sensitivity analyses were undertaken considering perturbations about the proposed staffing profiles under three scenarios: well match bed and theatre capacity; beds > theatres; theatres > beds. 25 perturbations of the staffing profiles were examined considered for each scenario; although there were a number of equivalent profiles, none offered any improvement in performance. 


\section{Validation of the simulation}

Comparisons of the model's key outputs and the observed ICU behaviour suggested that the model was a reasonable basis for management decision making. In particular, there was sufficient agreement in the measures of bed occupancy. The simulated mean occupancy was $15.4 \pm 0.2$, compared to an actual figure of 14.9. The mean occupancy is affected by assumptions about the starting conditions: the simulation reflects steady state behaviour and adopting a warm-up period to ensure a reasonable starting position; in reality, a series of changes at the HLC meant that this steady state assumption may not be completely true. The distribution of bed occupancy is illustrated in Fig.3. The deviations in the distributions may be explained by additional complexities in the management of the HLC. In reality there were fewer incidences of very high occupancy $(>19$ beds) than the simulation would suggest, with correspondingly more examples of medium occupancy. This could be evidence of a greater tendency to transfer patients out of the ICU to help maintain a contingency capacity. While it may well be useful to model this behaviour explicitly (Mallor \& Azcárate, 2011), insufficient data were available in the current study to provide any reliable insights.

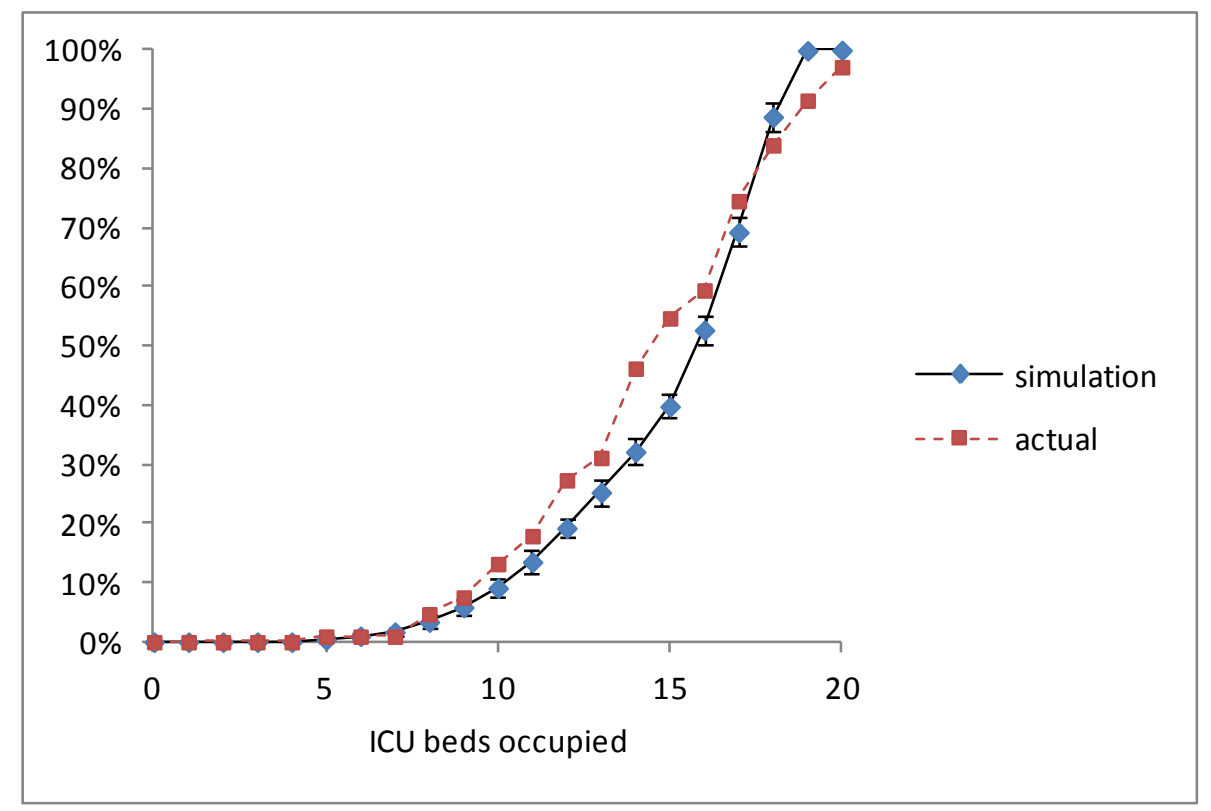

Fig.3 Comparing the cumulative distributions of simulated and actual bed occupancy

\section{Exploring resource utilisation}

A major concern in determining the capacity planning for the HLC was the utilisation of the expensive, scarce resources of ICU beds and operating theatre time. At the time of the study, it was generally believed that the ICU bed capacity was usually the constraining factor in the HLC and the immediate management questions were focussed on exploring options for increasing the ICU bed availability. The simulation was used to assess the possible benefits of increasing the ICU capacity, assuming that the practices of the HLC were unchanged with a theatre cancellation rate of $12 \%$ and a mean ICU length of stay of 3.7 days. In this 
simulation experiment the operating theatre capacity was kept constant with sufficient sessions for 30 patients per week assuming no cancellations; the total staffed ICU weekly bed capacity was varied from 78-128 bed-days adopting the proposed optimum staffing profile in each case. This range of ICU capacity considered in these simulation experiments was much greater than the practical options but it helped highlight some of the underlying relationships between the resources. Varying the ICU bed capacity, alters the bed:theatre balance. This balance was captured using a simple ratio $r$ of the potential bed capacity $(B)$, given the mean length of stay $(\mathrm{E}[s])$, and the daily theatre capacity $\left(t_{i}\right)$ incoporating the cancellation rate $(c)$ :

$$
r=\frac{B / \mathrm{E}[s]}{\sum_{i=1}^{7} t_{i}(1-c)}
$$

Fig.4 summarises the results of the experiment: when the bed and theatre capacity are well matched with an allocation of 97 bed-days per week and $r=0.997$, the mean HLC admissions were estimated to be $23.33 \pm 0.06$ per week; since the experiments considered a situation with substantial waiting lists, the admissions provided a measure of HLC capacity. Increasing ICU bed capacity, even with no complementary increase in theatre capacity, enhances the overall HLC capacity but with diminishing returns as noted in Fig.4.

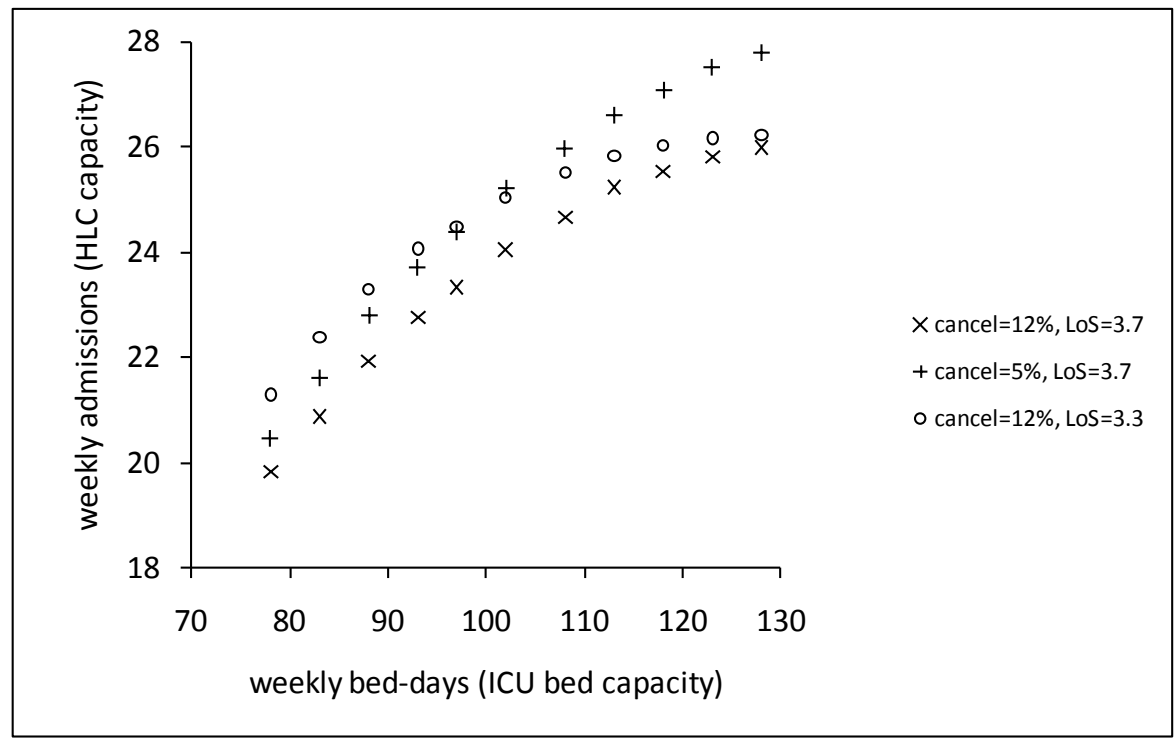

Fig.4 Diminishing returns of increasing bed capacity

As the bed capacity increases the theatres become the more frequent constraint and ICU beds are left vacant more often. Fig.5 captures this relationship between the utilisation of beds and theatres for the same sets of simulation experiments, n.b. the theatre utilisation is calculated as a proportion of the total available time and as such the maximum achievable is $(1-c)$. When there is a substantial surplus of operating theatre time, the utilisation of ICU beds is high; operations can be rescheduled in response to the likely bed availability as patients are transferred out of the ICU. However, if mean bed capacity exceeds the theatre capacity, it is 
more likely that there will be restricted flexibility in the theatre schedules to respond to variations in bed availability. When the capacities are well matched (weekly ICU capacity $=97$ bed-days; $r=0.997$ ), the simulation suggests that an ICU bed utilisation of $89 \%$ might be achievable, with a theatre utilisation of $89 \%$ $(1-c)=79 \%$. Alternative configurations with different balances of bed and theatre capacity will inevitably result in one resource being less well utilised. The extreme cases illustrate this trade-off: with an ICU capacity of just 78 bed-days per week $(r=0.802)$ the bed utilisation is $94 \%$, but the theatre utilisation falls to $66 \%$; increasing the ICU capacity to 128 bed-days per week $(r=1.315)$ would reduce bed utilisation to $75 \%$ but the theatre utilisation becomes $87 \%$.

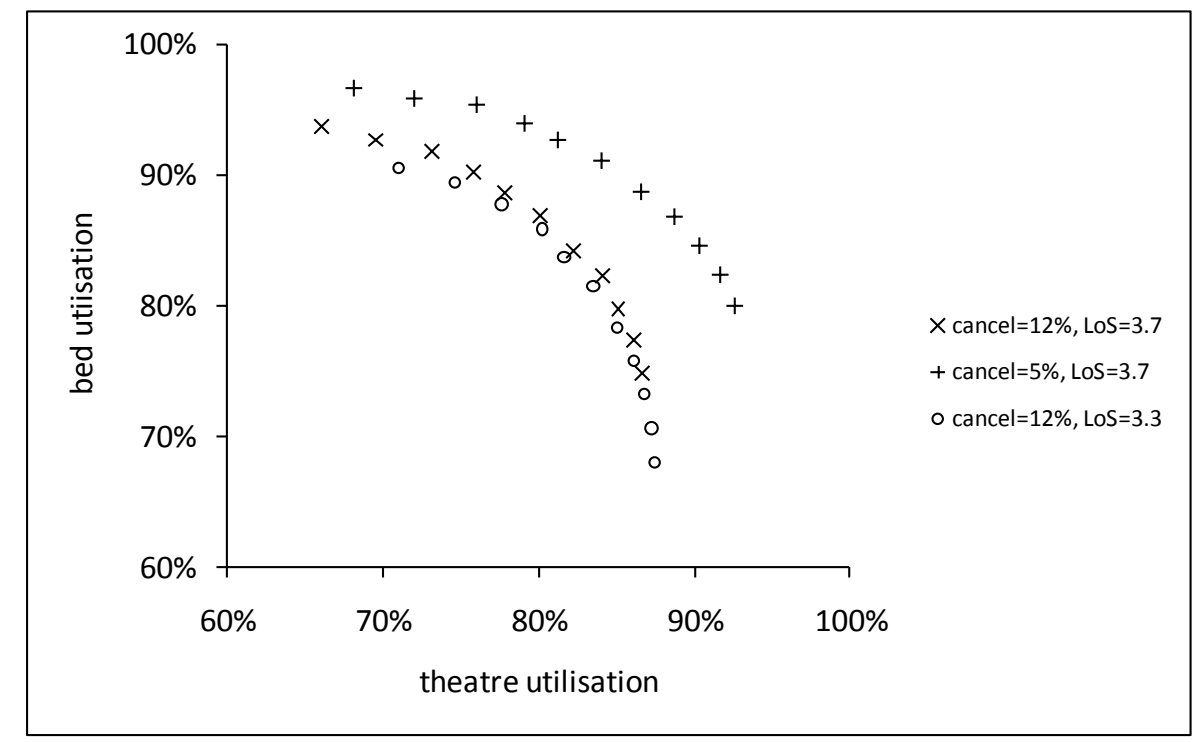

Fig. 5 The compromise between bed and theatre utilisation

Both operating theatre time and ICU beds are expensive, scarce resources. Typically the cost of surgery at the HLC is similar to the cost of ICU care (ISD Scotland, 2011) and Fig.6 depicts the combined ICU bed and theatre utilisation assuming equal weighting of the two resources. In this case, the simple measure of the bed:theatre ratio $r$ defined in Eq. (6) provides a good guide to the optimum configuration of theatres and beds with ICU with a weekly capacity of 97 beddays offering an overall HLC utilisation of $84 \%$, assuming an equal weighting for theatre time and ICU beds. The simulation experiments emphasise the relative insensitivity of the overall HLC utilisation to the ICU bed capacity. As long as the bed capacity is within $\pm 20 \%$ of the configuration suggested by Eq. 5 , the HLC utilisation remains above $80 \%$ : any change in ICU bed utilisation is largely compensated by the theatre utilisation. In other facilities the relative costs of surgery and bed-stay may well be different, reflected in a different weighting of theatre time and ICU beds in the overall HLC utilisation. Fig.6 illustrates the effects of different cost assumptions: if the typical HLC patients' surgery costs twice as much as the ICU stay, the balance shifts since it is important to try to make full use of the more expensive theatre time and a larger ICU is appropriate. The converse is also true: if the ICU stay were the more expensive component of the HLC care, the overall utilisation would be optimised with a smaller ICU. The simple measure of beds:theatres of Eq.5 is a useful guide to balancing the resource capacities when the resource costs are equal, otherwise more sophisticated 
models, such as the simulation of this study, are necessary if the optimal balance is required.

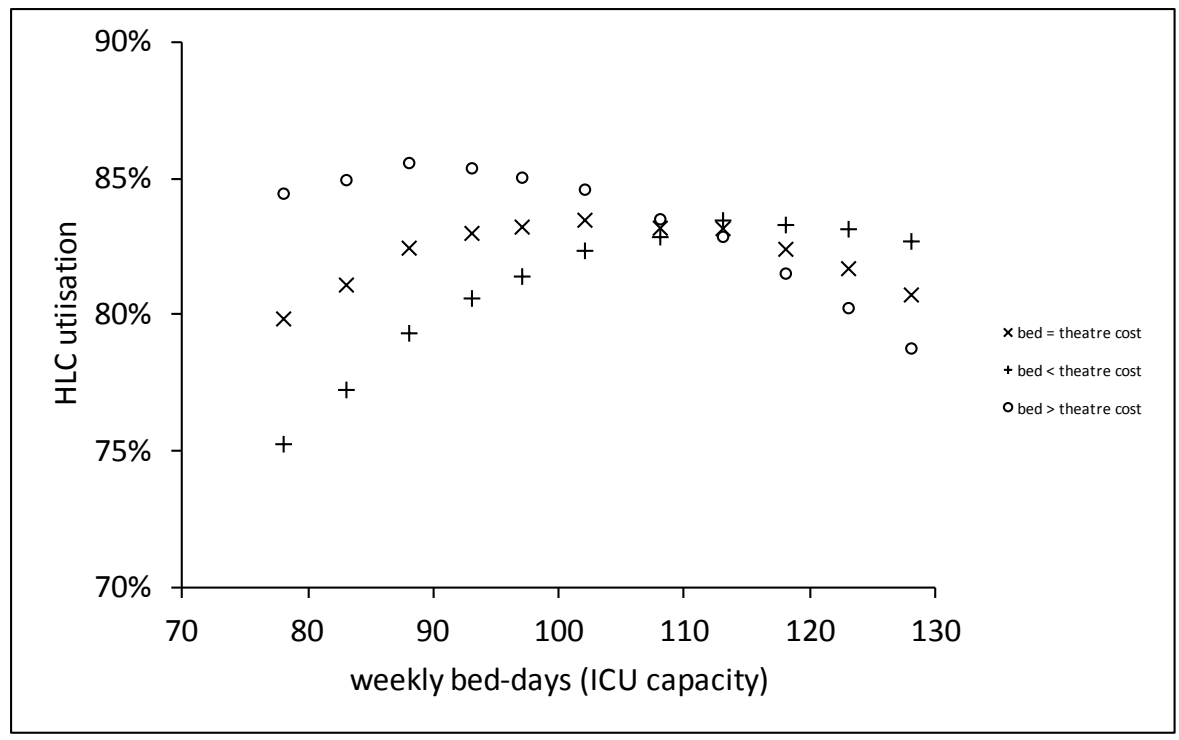

Fig.6 Optimising HLC utilisation

But is an optimal balance necessary? When bed:theatre cost $=0.5$, the simulation suggests that the overall HLC utilisation is optimised with a weekly capacity of 113 ICU bed-days, compared to the 97 implied by the simple measure $r$, see Eq. 6. This provides a $2.1 \%$ increase in HLC utilisation, as noted in Table 3 . If bed:theatre cost $=2.0$, the simulation suggests an optimal ICU capacity of 88 beddays, providing an increase of $0.5 \%$ in HLC utilisation. Even when the bed and theatre costs are not identical, the overall utilisation is relatively robust due to the trade-offs of the component utilisations illustrated in Fig.5. While the improvements in the HLC utilisations are statistically significant, as indicated by the $95 \%$ confidence intervals included in Table 3, the increases may not always be of great practical relevance. Utilisation is just one performance metric: HLC capacity may be more important. Furthermore, the options available to HLC management can be limited by operational resourcing practicalities, such as the scope to recruit qualified staff and the physical space available. However, presentations of the results of various sets of simulation experiments, such as Figs. 4-6, helped develop management's understanding of the inter-relationships between resources and identify a practical plan to increase the HLC capacity.

Table 3 Identifying the optimal balance (* ICU capacity suggested by the simulations)

\begin{tabular}{cccc}
\hline $\begin{array}{c}\text { bed:theatre } \\
\text { cost }\end{array}$ & $\begin{array}{c}B=\text { weekly } \\
\text { ICU capacity }\end{array}$ & $\begin{array}{c}r=\text { bed:theatre } \\
\text { capacity }\end{array}$ & $\begin{array}{c}\text { HLC } \\
\text { utilisation }\end{array}$ \\
\hline 0.5 & $113^{*}$ & 1.161 & $83.5 \pm 0.1 \%$ \\
0.5 & 97 & 0.997 & $81.4 \pm 0.2 \%$ \\
1.0 & $102^{*}$ & 1.048 & $83.5 \pm 0.2 \%$ \\
1.0 & 97 & 0.997 & $83.2 \pm 0.2 \%$ \\
2.0 & $88^{*}$ & 0.904 & $85.6 \pm 0.2 \%$ \\
2.0 & 97 & 0.997 & $85.1 \pm 0.2 \%$ \\
\hline
\end{tabular}




\section{Utilisation and the interaction of resources' availabilities}

One approach to increasing the HLC capacity is investment in additional beds or theatres but the model was also used to explore other possible management actions to enhance capacity. Analyses of the causes of theatre cancellations suggested that some might be avoidable. Improved theatre management can increase theatre availability: reducing the theatre cancellation rate to 5\% increases the overall HLC capacity as noted in Fig.4. This is especially valuable when the bed capacity is high and it is relatively easy to utilise the additional theatre sessions. Other actions focussed on the use of the ICU and the possibility of reducing the length of stay for some patients: this increases the ICU bed availability with a corresponding increase in HLC admissions, as illustrated in Fig. 4 by the results of the simulation experiment considering a reduction of $10 \%$ in the mean length of stay to 3.3 days. However, the increases in HLC capacity may not always be as great as expected since changing the availability of just one resource alters the beds: theatres balance, and the resource utilisations as reflected in Fig.5.

Where a single resource dominates, a simple trade-off between capacity and utilisation will typically emerge; increases in capacity will tend to be rewarded with diminishing increases in throughput. However, in the case of the HLC the overall capacity was determined by the combination of the availabilities of two symbiotic resources: beds and theatres. Increasing the number of beds, without increasing the theatre availability, will result in greater competition for theatre time and a reduction in bed utilisation. Failing to manage the ratio of beds:theatres will result in one of the resources being under utilised; it is important to consider the whole HLC system rather than focussing on the performance of one component resource.

\section{Management guidance}

Simple comparisons of high level statistics such as admissions and bed occupancy confirmed that the simulation provided a useful approximation to reality and a reasonable basis for capacity planning. A simulation model, with a simplified interface, was delivered for NHS staff use to explore various scenarios describing the possible development of the HLC. In addition to the model, some simple, general guidance was provided summarising the understanding developed from the simulation experiments:

- improvements in the efficient use of resources (e.g. shorter ICU bed stay or fewer theatre cancellations) are always beneficial;

- investing in additional capacity in either resource will enhance the overall system capacity but the benefits are greater if the resources are reasonably well matched;

- increasing the availability of just one resource will result in reducing its utilisation but increasing the utilisation of the companion resource;

- the optimum balance between the resources should reflect their relative costs. 


\section{Conclusions}

This study illustrates a capacity planning problem in which the daily demand is partly dependent on supply: the bed demand for any given day is a function of both the admissions on that day and also the admissions of previous days, which in turn are dependent on the bed availability for those days. An approximation ignoring these interactions facilitated an analytic formulation, further simplified by the use of an order 3 hyperexponential model of length of stay on the ICU. In this study, the approximation provided a reasonable estimate of relative performance and a basis for comparing options. This was sufficient to identify near optimal practice, such as efficient weekly staffing profiles. However, ignoring the interactions can produce significant errors in the estimates of the absolute capacity; a simulation was required to capture the interdependencies of the ICU beds and theatres, developing a fuller understanding of the subtle interactions and providing a more reliable approach to capacity planning for the whole system.

\section{References}

1. Adan, I., Bekkers, J., Dellaert, N., Jeunet, J., \& Vissers, J. (2011). Improving operational effectiveness of tactical master plans for emergency and elective patients under stochastic demand and capacitated resources. European Journal of Operational Research, 213, 290-308.

2. Blake, J. T. \& Donald, J. (2002). Mount Sinai Hospital uses integer programming to allocate operating room time. Interfaces, 32, 63-73.

3. Costa, A. X., Ridley, S. A., Shahani, A. K., Harper, P. R., De Senna, V., \& Nielsen, M. S. (2003). Mathematical modelling and simulation for planning critical care capacity. Anaesthesia, 58, 320-327.

4. Ernst, A. T., Jiang, H., Krishnamoorthy, M., \& Sier, D. (2004). Staff scheduling and rostering: A review of applications, methods and models. European Journal of Operational Research, 153, 3-27.

5. Fackrell, M. (2009). Modelling healthcare systems with phase-type distributions. Health Care Management Science, 12, 11-26.

6. Griffiths, J. D., Jones, M., Read, M. S., \& Williams, J. E. (2010). A simulation model of bed-occupancy in a critical care unit. Journal of Simulation, 4, 5259.

7. Griffiths, J. D., Price-Lloyd, N., Smithies, M., \& Williams, J. E. (2005). Modelling the requirement for supplementary nurses in an intensive care unit. Journal of the Operational Research Society, 56, 126-133.

8. Guerriero F., Guido R. (2011) Operational research in the management of the operating theatre: a survey, Health Care Management Science, 14, 89-114

9. Harper, P. R., Knight, V. A., \& Marshall, A. H. (2012). Discrete Conditional Phase-type models utilising classification trees: Application to modelling health service capacities. European Journal of Operational Research, 219, 522-530.

10. Heldwein, M., Badreldin, A., Doerr, F., Lehmann, T., Bayer, O., Doenst, T. \& Hekmat, K. (2011). Logistic Organ Dysfunction Score (LODS): A reliable postoperative risk management score also in cardiac surgical patients? Journal of Cardiothoracic Surgery, 6, 110. 
11. Ingolfsson, A., manul Haque, M., \& Umnikov, A. (2002). Accounting for time-varying queueing effects in workforce scheduling. European Journal of Operational Research, 139, 585-597.

12. ISD Scotland (2011). Costs Book 2010. Available at: http://www.isdscotlandarchive.scot.nhs.uk/isd/4683.html, accessed 20.9.11

13. Kim, S. C., Horowitz I., Young, K.K. \& Buckley, T.A. (1999). Analysis of capacity management of the intensive care unit in a hospital. European Journal of Operational Research, 115, 36-46.

14. Kim, S. C., Horowitz I., Young, K.K. \& Buckley, T.A. (2000). Flexible bed allocation and performance in the intensive care unit. Journal of Operations Management, 18, 427-443.

15. Kolker, A. (2009). Process Modeling of ICU Patient Flow: Effect of Daily Load Leveling of Elective Surgeries on ICU Diversion. Journal of Medical Systems, 33, 27-40.

16. Litvak, N., van Rijsbergen, M., Boucherie, R. J., \& van Houdenhoven, M. (2008). Managing the overflow of intensive care patients. European Journal of Operational Research, 185, 998-1010.

17. Mallor, F. \& Azcárate C. (2011) . Combining optimization with simulation to obtain credible models for intensive care units. Annals of Operations Research doi 10.1007/s10479-011-1035-8

18. Marshall, A. \& McClean, S. (2004). Using Coxian Phase-Type Distributions to Identify Patient Characteristics for Duration of Stay in Hospital. Health Care Management Science, 7, 285-289.

19. Marshall A.H \& Zenga M. (2012). Experimenting with the Coxian PhaseType Distribution to Uncover Suitable Fits. Methodology and Computing in Applied Probability, 14, 71-86.

20. McClean, S. \& Millard, P. (1993). Patterns of Length of Stay After Admission in Geriatric Medicine: An Event History Approach. Journal of the Royal Statistical Society. Series D (The Statistician), 42, 263-274.

21. McManus, M. L., Long, M. C., Cooper, A., \& Litvak, E. (2004). Queuing theory accurately models the need for critical care resources. Anesthesiology, 100, 1271-1276.

22. Morton, D. P. \& Popova, E. (2004). A Bayesian stochastic programming approach to an employee scheduling problem. Iie Transactions, 36, 155-167.

23. Oddoye, J. P., Jones, D. F., Tamiz, M., \& Schmidt, P. (2009). Combining simulation and goal programming for healthcare planning in a medical assessment unit. European Journal of Operational Research, 193, 250-261.

24. Ridge, J. C., Jones, S. K., Nielsen, M. S., \& Shahani, A. K. (1998). Capacity planning for intensive care units. European Journal of Operational Research, 105, 346-355.

25. Shahani, A. K., Ridley, S. A., \& Nielsen, M. S. (2008). Modelling patient flows as an aid to decision making for critical care capacities and organisation. Anaesthesia, 63, 1074-1080.

26. Shaw, B. \& Marshall, A. H. (2007). Modelling the flow of congestive heart failure patients through a hospital system. Journal of the Operational Research Society, 58, 212-218.

27. Sherck, J. \& Shatney, C. (1996). ICU scoring systems do not allow prediction of patient outcomes or comparison of ICU performance. Critical Care Clinics, 12, 515-523.

28. Siferd, S. P. \& Benton, W. C. (1994). A decision modes for shift scheduling of nurses. European Journal of Operational Research, 74, 519-527. 
29. Smith-Daniels, V. L., Schweikhart, S. B., \& Smith-Daniels, D. E. (1988). Capacity Management in Health Care Services: Review and Future Research Directions. Decision Sciences, 19, 889-919.

30. Utley, M., Gallivan, S., Treasure, T., \& Valencia, O. (2003). Analytical Methods for Calculating the Capacity Required to Operate an Effective Booked Admissions Policy for Elective Inpatient Services. Health Care Management Science, 6, 97-104.

31. Vanberkel, P. T., Boucherie, R. J., Hans, E. W., Hurink, J. L., van Lent, W. A. M., \& van Harten, W. H. (2011). An exact approach for relating recovering surgical patient workload to the master surgical schedule. Journal of the Operational Research Society, 62, 1851-1860.

32. Vasilakis, C. \& Marshall, A. H. (2005). Modelling nationwide hospital length of stay: opening the black box. Journal of the Operational Research Society, 56, 862-869.

33. Vassilacoupolos, G. (1985). Allocating Doctors to Shifts in an Accident and Emergency Department. Journal of the Operational Research Society, 36, 517-523.

34. Vissers, J. (1998). Patient flow-based allocation of inpatient resources: a case study. European Journal of Operational Research, 105, 356-370. 